\title{
PARTIAL PROLONGATIONS AND CHARACTERISTICS OF DIFFERENTIAL EQUATIONS
}

\author{
BY
}

H. H. JOHNSON

This paper studies the behavior of characteristics under partial prolongations. Partial prolongations define a natural equivalence relation between systems of partial differential equations [2], [3]. Examples are given to show that characteristics are not preserved in general. They are, however, preserved in an important case, namely, in determined or over-determined systems the highest-dimensional characteristics are proved to be invariant. A further example shows that even in determined systems lower-dimensional characteristics are not preserved.

1. Definitions. We take the viewpoint of E. Cartan in [1], where he very clearly defined involutiveness and characteristics for quasilinear systems, rather than his usual systems of exterior differential forms [2], [5].

All functions, maps and manifolds are infinitely differentiable. "Function" means real-valued function unless otherwise noted. Let the manifold $V$ be fibred over the manifold $U$ by $\pi: V \rightarrow U$. If $\pi(v)=u$, one may choose fibred coordinates $x^{1}, \ldots, x^{p}$ around $u$ and $x^{1}, \ldots, x^{p}, z^{1}, \ldots, z^{m}$ around $v$ so that $x^{i} \pi=x^{i}$, all $i$. We may denote $x^{i}$ by $x^{i}$ without confusion. The $x^{i}$ are called independent, the $z^{\lambda}$ the dependent, variables. Let $J^{1}=J^{1}(U, V)$ denote the 1 -jets $j_{x}^{1}(s)$ of sections $s: U \rightarrow V$ satisfying $\pi s=$ identity. When $\left(x^{i}, z^{\lambda}\right)$ are fibred coordinates on $V, J^{1}$ will have fibred coordinates $\left(x^{i}, z^{\lambda}, \partial_{i} z^{\lambda}\right)$ where $\partial_{i} z^{\lambda}=\left(\partial s^{\lambda} / \partial x^{i}\right)(x), \quad \lambda=1, \ldots, m$; $i=1, \ldots, p$ (these will be the usual ranges of $\lambda$ and $i$ ). We shall study functions on $J^{1}$ which are linear in $\partial_{i} z^{\lambda}: A_{\lambda}^{i}(x, z) \partial_{i} z^{\lambda}+B(x, z)$. (We use the summation convention.) The property of being linear in $\partial_{i} z^{\lambda}$ is invariant under local fibred coordinate changes, and such functions define quasilinear partial differential equations $A_{\lambda}^{i}\left(\partial z^{\lambda} / \partial x^{i}\right)+B=0$. We study a collection $\Sigma$ of such functions on $J^{1}$ which form a finitely-generated module over the ring of functions on $V$, thus a system of quasilinear partial differential equations, or a system.

A solution of $\Sigma$ is a section $s: U \rightarrow V, \pi s=$ identity, such that $f\left(j_{x}^{1}(s)\right)=0$ for all $x$ in $U$ and all $f$ in $\Sigma$. A jet $j_{x}^{1}(s)$ is integral for $\Sigma$ if all functions in $\Sigma$ vanish at $j_{x}^{1}(s)$. Two systems are equivalent if they define the same set of integral jets. If $S$ is the set of integral jets, $\beta(S)$ is the set of integral points in $V$, where $\beta$ is the usual target map $\beta\left(j_{x}^{1}(s)\right)=s(x)$. 
When the coefficients $A_{\lambda}^{i}, B$ of the functions in $\Sigma$ are evaluated at an integral point $\left(x_{0}, z_{0}\right)$ in $V, \Sigma$ becomes a subspace $\Sigma^{0}$ of the $(m p+1)$-dimensional vector space of polynomials in $\hat{\partial}_{i} z^{\lambda}$ of order at most 1 over $R$ (real numbers). Let $\Sigma_{h}^{0}$ be the subspace of such polynomials which depend only on $\partial_{1} z^{\lambda}, \ldots, \partial_{h} z^{\lambda}$.

Let $r_{h}=\operatorname{dim} \Sigma_{h}^{0}-\operatorname{dim} \Sigma_{h-1}^{0}$. We call the coordinates $x^{1}, \ldots, x^{p}$ on $U h$-nonsingular at $\left(x_{0}, z_{0}\right)$ if, when $h=p, r_{p}$ is maximal among all independent variables, and, when $h<p, r_{h}$ is maximal among all $(h+1)$-nonsingular independent variables. Also 1-nonsingular variables at $\left(x_{0}, z_{0}\right)$ are called nonsingular at $\left(x_{0}, z_{0}\right)$. These definitions only depend on the tangent vectors to the coordinate curves in $U$ at $\left(x_{0}\right)$, hence there exist nonsingular independent variables at $\left(x_{0}, z_{0}\right)$ and they are dense and open in the Stiefel manifold of frames at $\left(x_{0}\right)$. Let $r_{h}\left(x_{0}, z_{0}\right)$ be the value of $r_{h}$ for nonsingular independent variables. Observe that $r_{h+1}\left(x_{0}, z_{0}\right) \geqq r_{h}\left(x_{0}, z_{0}\right)$. We shall assume that these integer-valued functions are constant on $V$.

To each $\alpha=\left(\alpha_{1}, \ldots, \alpha_{p}\right)$ in $R^{p}$, to each $A_{\lambda}^{i} \partial_{i} z^{\lambda}+B$ in $\Sigma$ and to each integral point $\left(x_{0}, z_{0}\right)$ in $V$ we associate a linear form $A_{\lambda}^{i}\left(x_{0}, z_{0}\right) \alpha_{i} \xi^{\lambda}=F\left(\xi^{1}, \ldots, \xi^{m}\right)$ on $R^{m}$. Let $\Sigma_{f}\left(x_{0}, z_{0}, \alpha\right)$ be the vector space of such forms.

Proposition 1. Independent variables $x^{1}, \ldots, x^{p}$ are p-singular at $\left(x_{0}, z_{0}\right)$ if and only if $\operatorname{dim} \Sigma_{f}\left(x_{0}, z_{0}, \alpha\right)<r_{p}\left(x_{0}, z_{0}\right)$, where $\alpha_{j}=\left(\partial \boldsymbol{x}^{p} / \partial x^{j}\right)\left(x_{0}\right)$.

Proof. If $x^{i}$ are nonsingular at $\left(x_{0}, z_{0}\right)$ and $\Sigma$ is generated by $f^{\alpha}=A_{\lambda}^{\alpha j} \partial_{j} z^{\lambda}+B$, $\alpha=1, \ldots, \alpha_{t}$, then $r_{p}\left(x_{0}, z_{0}\right)=\operatorname{rank}\left(A_{\lambda}^{\alpha p}\left(x_{0}, z_{0}\right)\right)$. For other variables $x^{i}, z^{\lambda}, \partial_{i} \bar{z}^{\lambda}$, $f^{\alpha}=\bar{A}_{\lambda}^{\alpha j} \partial_{j} \bar{z}^{\lambda}+B$ where $\bar{A}_{\lambda}^{\alpha j}=A_{\lambda}^{\alpha k}\left(\partial x^{j} / \partial x^{k}\right)$, hence

$$
r_{p}=\operatorname{rank}\left(\bar{A}_{\lambda}^{\alpha p}\left(x_{0}, z_{0}\right)\right)=\operatorname{dim} \Sigma_{f}\left(x_{0}, z_{0}, \alpha\right)
$$

when $\alpha_{i}=\left(\partial x^{p} / \partial x^{i}\right)\left(x_{0}\right)$.

Geometrically, $\alpha_{1}, \ldots, \alpha_{p}$ define a $(p-1)$-dimensional subspace in the tangent space to $U$ at $x_{0}$ consisting of all vectors $a^{i}\left(\partial / \partial x^{i}\right)$ where $a^{i} \alpha_{i}=0$. Independent variables $x^{i}$ are $p$-singular at $\left(x_{0}, z_{0}\right)$ when $\partial / \partial x^{1}, \ldots, \partial / \partial x^{p-1}$ span a subspace defined by $\alpha$ where $\operatorname{dim} \Sigma_{f}\left(x_{0}, z_{0}, \alpha\right)<r_{p}\left(x_{0}, z_{0}\right)$.

Definition 1. A $(p-1)$-dimensional subspace of the tangent space to $U$ at $x_{0}$ is $(p-1)$-characteristic for $\Sigma$ at $\left(x_{0}, z_{0}\right)$ if it is defined by $\alpha_{1}, \ldots, \alpha_{p}$ where

$$
\operatorname{dim} \Sigma_{f}\left(x_{0}, z_{0}, \alpha\right)<r_{p}\left(x_{0}, z_{0}\right) .
$$

In the same way, independent variables $x^{i}$ are $(p-2)$-singular at $\left(x_{0}, z_{0}\right)$ when the system of forms $A_{\lambda}^{\alpha j}\left(x_{0}, z_{0}\right)\left(\alpha_{j} \xi^{\lambda}+\beta_{j} \eta^{\lambda}\right)=F\left(\xi^{1}, \ldots, \xi^{m}, \eta^{1}, \ldots, \eta^{m}\right)$ has

$$
\text { rank }<r_{p}\left(x_{0}, z_{0}\right)+r_{p-1}\left(x_{0}, z_{0}\right) \text {, }
$$

where $\alpha_{j}=\left(\partial x^{p} / \partial x^{j}\right)\left(x_{0}\right)$ and $\beta_{j}=\left(\partial x^{p-1} / \partial x^{j}\right)\left(x_{0}\right)$. A $(p-2)$-dimensional subspace of the tangent space to $U$ at $x_{0}$ is characteristic for $\Sigma$ at $\left(x_{0}, z_{0}\right)$ if it consists in all vectors $a^{i}\left(\partial / \partial x^{i}\right)$ satisfying $a^{i} \alpha_{i}=a^{i} \beta_{i}=0$ where the forms $F^{\alpha}$ have

$$
\text { rank }<r_{p}\left(x_{0}, z_{0}\right)+r_{p-1}\left(x_{0}, z_{0}\right) \text {. }
$$


In a similar way the other lower-dimensional characteristics are defined. Two equivalent systems have the same characteristics.

Involutiveness of our systems may be defined as follows. Let $J^{2}(U, V)$ be the 2-jets of sections with coordinates $\left(x^{i}, z^{\lambda}, \partial_{i} z^{\lambda}, \partial_{i j} z^{\lambda}\right)$ where $\partial_{i j} z^{\lambda}=\partial_{j i} z^{\lambda} ; i, j=$ $1, \ldots, p ; \lambda=1, \ldots, m$. If $f$ is any function on $J^{1}$, let $\partial_{j}^{*} f=\left(\partial f / \partial x^{j}\right)+\left(\partial f / \partial z^{\lambda}\right) \partial_{j} z^{\lambda}$ $+\left(\partial f / \partial\left(\partial_{i} z^{\lambda}\right)\right) \partial_{i j} z^{\lambda}$. For a system $\Sigma$, we let $\partial_{j}^{*} \Sigma$ be the module generated by $\left\{\partial_{j}^{*} f: f \in \Sigma\right\}$, considered as a module over the functions on $J^{1}$. If $\left(x_{0}^{i}, z_{0}^{\lambda}, \partial_{i} z_{0}^{\lambda}\right)$ $=\left(x_{0}, z_{0}, \partial z_{0}\right)$ is in $J^{1},\left(\partial_{j}^{*} \Sigma\right)^{0}$ denotes the elements of $\partial_{j}^{*} \Sigma$ evaluated at $\left(x_{0}, z_{0}, \partial z_{0}\right)$. These are linear polynomials over $R$ in $\partial_{i j} z^{\lambda}$.

Definition 2. The system $\Sigma$ is involutive at an integral jet $\left(x_{0}, z_{0}, \partial z_{0}\right)$ if for some independent variables nonsingular at $\left(x_{0}, z_{0}\right)$, it happens that for every $h$ where $0 \leqq h \leqq p-1, \quad 1 \leqq k \leqq p-h, \quad\left(\partial_{h+k}^{*} \Sigma_{h}\right)^{0} \subset\left(\partial_{1}^{*} \Sigma_{h+k}\right)^{0}+\cdots+\left(\partial_{h}^{*} \Sigma_{h+k}\right)^{0}+\Sigma_{h+k}^{0}$ where $A+B$ means the vector space generated by $A$ and $B$ in the linear polynomials over $R$ in $\partial_{i j} z^{\lambda}$. We call $\Sigma$ involutive if it is involutive at all integral jets.

Intuitively, involutiveness means that no information about $\Sigma$ can be gained by equating mixed partials. E. Cartan proved that involutiveness is equivalent to the condition that $\left(\partial_{1}^{*} \Sigma\right)^{0}+\cdots+\left(\partial_{p}^{*} \Sigma\right)^{0}$ has dimension $p r_{p}-\sum_{h=1}^{p-1}(p-h)\left(r_{h}-r_{h-1}\right)+1$, and that it does not depend on the particular choice of nonsingular independent variables $x^{i}[1]$. We shall assume all systems to be involutive.

Partial prolongations are defined in terms of total prolongations which may be defined as follows. Let $V_{0}$ now denote $J^{1}$ with coordinates $\left(x^{i}, z^{\lambda}, p_{i}^{\lambda}\right)$ and let $\pi_{0}: V_{0} \rightarrow U$ be the obvious fibre map. Consider $J^{1}\left(U, V_{0}\right)$ with coordinates $\left(x^{i}, z^{\lambda}, p_{i}^{\lambda}, \partial_{i} z^{\lambda}, \partial_{i} p_{j}^{\lambda}\right)$. There is a natural imbedding $\phi: J^{2}(U, V) \rightarrow J^{1}\left(U, V_{0}\right)$ defined by $\phi\left(j_{x}^{2}(s)\right)=j_{x}^{1}\left(j^{1}(s)\right)$, i.e., $\phi\left(x^{i}, z^{\lambda}, \partial_{i} z^{\lambda}, \partial_{i j} z^{\lambda}\right)=\left(x^{i}, z^{\lambda}, \partial_{i} z^{\lambda}, \partial_{i} z^{\lambda}, \partial_{i j} z^{\lambda}\right)$, so $\phi\left(J^{2}\right)$ is the submanifold of $J^{1}\left(U, V_{0}\right)$ defined by $\partial_{i} z^{\lambda}=p_{i}^{\lambda}, \partial_{i} p_{j}^{\lambda}=\partial_{j} p_{i}^{\lambda}$. Given a function $f$ on $V_{0}$ we define $\partial_{j}^{\#} f$ on $J^{1}\left(U, V_{0}\right)$ by

$$
\partial_{i}^{\#} f=\left(\partial f / \partial x^{i}\right)+\left(\partial f / \partial z^{\lambda}\right) p_{i}^{\lambda}+\left(\partial f / \partial p_{j}^{\lambda}\right) \partial_{i} p_{j}^{\lambda}
$$

so that $\partial_{i}^{*} f=\left(\partial_{i}^{\#} f\right) \phi$. As above, we can define $\partial_{i}^{\#} \Sigma$ for any system $\Sigma$, and $P \Sigma$ is the system on $J^{1}\left(U, V_{0}\right)$ generated by $\partial_{1}^{\#} \Sigma+\cdots+\partial_{p}^{\#} \Sigma+\Sigma$ together with $\partial_{i} z^{\lambda}-p_{i}^{\lambda}$ and $\partial_{i} p_{j}^{\lambda}-\partial_{j} p_{i}^{\lambda}$ as a module over the functions on $V_{0}$. This system $P \Sigma$ is the total prolongation of $\Sigma$, abbreviated tp.

If $s: U \rightarrow V$ is a solution of $\Sigma$, then $j^{1}(s)$ is a solution of $P \Sigma$, and all solutions of $P \Sigma$ arise in this way. The involutiveness of $\Sigma$ (in fact the conditions $\left(\partial_{k}^{*} \Sigma_{0}\right)^{0}$ $\left.\subset\left(\Sigma_{k}\right)^{0}\right)$ imply that any function $f_{0}$ on $V_{0}$ which occurs in $P \Sigma$ must vanish on the integral jets of $\Sigma$.

REMARK. Total prolongations can be invariantly defined for much more general systems of partial differential equations [5], but the result is always a quasilinear system. Hence it is not a serious restriction to consider only quasilinear systems.

Definition 3. A partial prolongation (abbreviated pp) of $\Sigma$ is a system $\Sigma^{\prime}$ on a fibred manifold $V_{1}, \pi_{1}: V_{1} \rightarrow U$ satisfying the following conditions: 
(1) There is a fibration $\rho_{0}: V_{1} \rightarrow V$, and $\pi \rho_{0}=\pi_{1}$. If $\rho: J^{1}\left(U, V_{1}\right) \rightarrow J^{1}(U, V)$ is $\rho\left(j_{x}^{1}\left(s_{1}\right)\right)=j_{x}^{1}\left(\rho_{0} s_{1}\right)$, then $f \rho$ is in $\Sigma^{\prime}$ for every $f$ in $\Sigma$.

(2) $V_{0}=J^{1}(U, V)$ is fibred over $V_{1}$ by some $\sigma_{0}: V_{0} \rightarrow V_{1}$ such that $\rho_{0} \sigma_{0}=\beta$, the target projection in jets. If $\sigma: J^{1}\left(U, V_{0}\right) \rightarrow J^{1}\left(U, V_{1}\right)$ is $\sigma\left(j_{x}^{1}\left(s_{0}\right)\right)=j_{x}^{1}\left(\sigma_{0} s_{0}\right)$, then $f_{1} \sigma$ is in $P \Sigma$ for every $f_{1}$ in $\Sigma^{\prime}$.

(3) For any point $v_{1}$ in $V_{1}$ and $r=\pi\left(v_{1}\right)$ in $V$ and for any coordinates $\left(x^{i}, z^{\lambda}\right)$ on $V$, it is possible to choose linearized coordinates $\left(x^{i}, z^{\lambda}, u^{\pi}\right)$ on $V_{1}$ near $v_{1}$ so that $\sigma_{0}$ is linear in $p_{j}^{\lambda}: \sigma_{0}\left(x^{i}, z^{\lambda}, p_{i}^{\lambda}\right)=\left(x^{i}, z^{\lambda}, K_{\lambda}^{\pi j}(x, z) p_{j}^{\lambda}+L^{\pi}(x, z)\right)$, where the rank of $\left(K_{\lambda}^{\pi j}\right)$ is constant. If $p$ is any integral jet of $\Sigma^{\prime}$, then it is required that $\sigma_{0} \rho(p)=\beta(p)$.

(4) For every integral jet $p$ of $\Sigma$ in $J^{1}(U, V)$, there is at least one integral jet $q$ of $\Sigma^{\prime}$ in $J^{1}\left(U, V_{1}\right)$ such that $\beta(q)=\sigma_{0}(p)$.

REMARK. The assumption of linearized coordinates in (3) is not as serious a restriction as it appears, for the same reason as the assumption of quasilinearity. For, given a more general $\mathrm{pp}$, taking the tp of everything yields a pp satisfying the linearized condition. Compare [3]. Condition (4) would not be necessary in the real analytic case, for then solutions $s$ exist through $p$, and $q=\sigma\left(j_{x}^{1}(s)\right)$.

Proposition 2. Let $\Sigma$ ' be a pp of $\Sigma$. If $s$ is a solution of $\Sigma$, then $\sigma_{0} j^{1}(s)$ is a solution of $\Sigma^{\prime}$. If $s_{1}$ is a solution of $\Sigma^{\prime}$, then $\rho_{0} s_{1}$ is a solution of $\Sigma$. Further, $\rho_{0} \sigma_{0} j^{1}(s)=s$, and $\sigma_{0} j^{1}\left(\rho_{0} s_{1}\right)=s_{1}$.

Proof. The first and second assertions follow at once from (1) and (2). By (2), $\rho_{0} \sigma_{0}\left(j_{x}^{1}(s)\right)=\beta\left(j_{x}^{1}(s)\right)=s(x)$. The last equation follows from (3).

Proposition 3. Let $\Sigma^{\prime}$ be a pp of $\Sigma$, and let $\left(x^{i}, z^{\lambda}, u^{\pi}\right)$ be linearized coordinates on a neighborhood $V_{1}^{\prime}$ in $V_{1}$ so that $\sigma_{0}\left(x^{i}, z^{\lambda}, p_{i}^{\lambda}\right)=\left(x^{i}, z^{\lambda}, K_{\lambda}^{\pi j} p_{j}^{\lambda}+L^{\pi}\right)$. Then $\Sigma^{\prime}$ is generated by $f \rho$, for $f$ in $\Sigma$, the functions $u^{\pi}-K_{\lambda}^{\pi j} \partial_{j} z^{\lambda}-L^{\pi}$ and a set of functions linearly independent in the $\partial_{i} u^{\pi}$.

Proof. In local linearized coordinates, suppose $\Sigma$ is generated by $f^{\alpha}=A_{\lambda}^{\alpha j} \partial_{j} z^{\lambda}+B^{\alpha}$ and suppose $\Sigma^{\prime}$ contains in addition the functions $g^{\sigma}=H_{\lambda}^{\sigma j} \partial_{j} z^{\lambda}+I^{\sigma}, \sigma=1, \ldots, \sigma_{t}$ maximally linearly dependent on $\partial_{i} z^{\lambda}$. Now by (2) if one of the $g^{\sigma}$ were a function of $x^{i}, z^{\lambda}, \partial_{i} z^{\lambda}$ only, it would appear in $P \Sigma$ as a function of $x^{i}, z^{\lambda}, p_{j}^{\lambda}$ only, hence would appear in $\Sigma$, i.e., be a function of the $f^{\alpha}$. We may suppose, then, that the $g^{o}$ are functions of $x^{i}, z^{\lambda}, \partial_{i} z^{\lambda}$ and $u^{\pi}$.

For every $\left(x^{i}, z^{\lambda}, u^{\pi}, \partial_{i} z^{\lambda}\right)$ satisfying $f^{\alpha}=0, g^{\sigma}=0$, it is possible to choose $\partial_{i} u^{\pi}$ to annihilate the remaining equations of $\Sigma^{\prime}$, since otherwise there must be more equations in $\Sigma$ independent of $\partial_{i} u^{\pi}$. Then by (3) $u^{\pi}=K_{\lambda}^{\pi j} \partial_{j} z^{\lambda}+L^{\pi}$ when $f^{\alpha}=g^{\sigma}=0$. By (4), if $\left(x^{i}, z^{\lambda}, \partial_{j} z^{\lambda}\right)$ satisfy $f^{\alpha}=0$, there is at least one set of values $u^{\pi}, \partial_{i} u^{\pi}$ satisfying $u^{\pi}=K_{\lambda}^{\pi j} \partial_{j} z^{\lambda}+L^{\pi}$ where the functions of $\Sigma^{\prime}$ vanish. Hence for $u^{\pi}=$ $K_{\lambda}^{\pi j}(x, z) \hat{\partial}_{j} z^{\lambda}+L^{\pi}(x, z)$, all $g^{\sigma}=0$. Thus the systems $\left\{A_{\lambda}^{\alpha j} \partial_{j} z^{\lambda}+B^{\alpha}, u^{\pi}-K_{\lambda}^{\pi j} \partial_{j} z^{\lambda}-L^{n}\right\}$ and $\left\{A_{\lambda}^{\alpha j} \partial_{j} z^{\lambda}+B^{\alpha}, H_{\lambda}^{\sigma j} \partial_{j} z^{\lambda}+I^{\sigma}\right\}$ have exactly the same annihilators.

Since we are studying characteristics it is not a serious restriction to consider only pp's $\Sigma^{\prime}$ on domains on which linearized fibred coordinates $\left(x^{i}, z^{\lambda}, u^{n}\right)$ exist 
globally and which contain the functions $u^{\pi}-K_{\lambda}^{\pi j} \partial_{j} z^{\lambda}-L^{\pi}$. This we shall do in the rest of this paper.

Proposition 4. Let $\Sigma^{\prime}$ be a pp of $\Sigma$ with $\left(x^{i}, z^{\lambda}, u^{\pi}\right)$ linearized coordinates on $V$, where $\sigma_{0}$ is defined by $u^{\pi}=K_{\lambda}^{\pi j} \partial_{j} z^{\lambda}+L^{\pi}$. If $G=R_{\pi}^{j} \partial_{j} u^{\pi}+S_{\lambda}^{j} \partial_{j} z^{\lambda}+T$ is in $\Sigma^{\prime}$, then $\boldsymbol{R}_{\pi}^{j} \partial_{j}^{*}\left(K_{\lambda}^{\pi k} \partial_{k} z^{\lambda}+L^{\pi}\right)+S_{\lambda}^{j} \partial_{j} z^{\lambda}+T$ is in $\partial^{*}(\Sigma)$, where $\boldsymbol{F}=F \sigma_{0}$ and $\partial^{*}(\Sigma)$ is the module of functions on $J^{2}(U, V)$ generated by $\left\{\partial_{j}^{*} f, f: f \in \Sigma\right\}$ over the ring of functions on $J^{1}(U, V)$.

Proof. By (2) of Definition $3 G \sigma$ is in $P \Sigma$, and

$$
\sigma\left(x^{i}, z^{\lambda}, p_{j}^{\lambda}, \partial_{j} z^{\lambda}, \partial_{j} p_{i}^{\lambda}\right)=\left(x^{i}, z^{\lambda}, K_{\lambda}^{\pi j} p_{j}^{\lambda}, K_{\lambda}^{\pi j} \partial_{i} p_{j}^{\lambda}+\left(\partial_{i}^{*} K_{\lambda}^{\pi j}\right) p_{j}^{\lambda}-\partial_{i}^{*} L^{\pi}\right) .
$$

If $\phi: J^{2}(U, V) \rightarrow J^{1}\left(U, V_{0}\right)$ is the canonical map $\phi\left(j_{x}^{2}(s)\right)=j_{x}^{1}\left(j^{1}(s)\right)$, it is merely a calculation to see that $G \sigma \phi$ has the form above and belongs to $P \Sigma \phi=\partial^{*}(\Sigma)$.

Proposition 5. Let $\Sigma$ be a system on $V$ and $\Sigma^{\prime}$ a system on $V_{1}=V \times W$ where $W$ is $\pi_{t}$-dimensional real space, and $V_{1}$ is fibred over $V$ by $\rho_{0}: V_{1} \rightarrow V$ as well as over $U$ by $\pi_{1}: V_{1} \rightarrow U$, and $\pi \rho_{0}=\pi_{1}$. Let $\left(x^{i}, z^{\lambda}, u^{\pi}\right)$ be local coordinates on $V_{1}$ where $\left(x^{i}, z^{\lambda}\right)$ are fibred coordinates on $V$. Assume that $\Sigma$ is generated by $A_{\lambda}^{\alpha j} \partial_{j} z^{\lambda}+B^{\alpha}$, and that $\Sigma^{\prime}$ is generated by $A_{\lambda}^{\alpha j} \partial_{j} z^{\lambda}+B^{\alpha}, u^{\pi}-K_{\lambda}^{\pi j} \partial_{j} z^{\lambda}-L^{\pi}, R_{\pi}^{\gamma j} \partial_{j} u^{\pi}+S_{\lambda}^{\gamma j} \partial_{j} z^{\lambda}+T^{\gamma}$ where

(a) $K_{\lambda}^{\pi j}$ and $L^{\pi}$ depend only on $x^{i}, z^{\lambda}$, and the Jacobian of $K_{\lambda}^{\pi j} \partial_{j} z^{\lambda}+L^{\pi}$ is of rank $\pi_{l}$;

(b) $\boldsymbol{R}_{\pi}^{\gamma j} \partial_{j}^{*}\left(K_{\lambda}^{\pi i} \partial_{i} z^{\lambda}+L^{\pi}\right)+S_{\lambda}^{\gamma j} \partial_{j} z^{\lambda}+T^{\gamma}$ is in $\partial^{*}(\Sigma)$, where if $F=F\left(x^{i}, z^{\lambda}, u^{\pi}\right)$, then $F=F\left(x^{i}, z^{\lambda}, K_{\lambda}^{\pi j} \partial_{j} z^{\lambda}+L^{\pi}\right)$;

(c) for every $\left(x^{i}, z^{\lambda}, u^{\pi}\right)$, rank $\left(R_{\pi}^{\gamma j}\right)=\gamma_{t}$; where $\gamma=1, \ldots, \gamma_{t}$ and $\alpha=1, \ldots, \alpha_{t}$.

Then $\Sigma^{\prime}$ is a pp of $\Sigma$.

Proof. Define $\sigma_{0}: V_{0} \rightarrow V_{1}$ by $\sigma_{0}\left(x^{i}, z^{\lambda}, p_{i}^{\lambda}\right)=\left(x^{i}, z^{\lambda}, K_{\lambda}^{\pi j} p_{j}^{\lambda}+L^{\pi}\right)$. In Definition 3 condition (1) is satisfied because $A_{\lambda}^{\alpha j} \partial_{j} z^{\lambda}+B^{\alpha}$ is in $\Sigma^{\prime}$. Condition (2) is satisfied because of hypothesis (b). Linearized coordinates exist by definition of $\sigma_{0}$. If $p=\left(x^{i}, z^{\lambda}, u^{\pi}, \partial_{j} z^{\lambda}, \partial_{j} u^{\pi}\right)$ is an integral jet of $\Sigma^{\prime}$,

$$
\sigma_{0} \rho(p)=\sigma_{0}\left(x^{i}, z^{\lambda}, \partial_{j} z^{\lambda}\right)=\left(x^{i}, z^{\lambda}, K_{\lambda}^{\pi j} \partial_{j} z^{\lambda}+L^{\pi}\right)=\left(x^{i}, z^{\lambda}, u^{\pi}\right)=\beta(p),
$$

since $u^{\pi}-K_{\lambda}^{\pi j} \partial_{j} z^{\lambda}-L^{\pi}=0$. Thus (3) is satisfied. For any $\left(x^{i}, z^{\lambda}, \partial_{i} z^{\lambda}\right)$ satisfying $A_{\lambda}^{\alpha j} \partial_{j} z^{\lambda}+B^{\alpha}=0$, there is $u^{\pi}=K_{\lambda}^{\pi j} \partial_{j} z^{\lambda}+L^{\pi}$ and we can solve $R_{\pi}^{\gamma j} \partial_{j} u^{\pi}+\cdots=0$. Hence (4) is satisfied.

Two systems $\Sigma$ and $\Sigma^{\prime}$ are absolutely equivalent if there is a sequence of systems $\Sigma, \Sigma_{1}, \Sigma_{2}, \ldots, \Sigma_{k}, \Sigma^{\prime}$ where for each adjacent pair one is the pp of the other. Properties which are unchanged by pp's are absolute invariants. In [1, p. 1134] E. Cartan described the relation of partial prolongation as "... le plus large de ceux qu'on peut actuellement soumettre à l'analyse et le seul du reste qui semble important dans les applications." In [4] it was shown that hyperbolicity is not an absolute invariant. Here we study absolute invariance of characteristics. 
Proposition 6. The $(p-1)$-characteristics of $P \Sigma$ at $q$ and of $\Sigma$ at $\beta(q)$ coincide.

Proof. This follows from a local coordinate representation and, for any $\left(\alpha_{j}\right)$, the observation that by a change of coordinates $x^{i}$ one can suppose $\left(\alpha_{j}\right)=\left(\delta_{j}^{1}\right)$.

There is one further way to modify systems. Given $\Sigma$ on $V$, if $V_{1}$ is a submanifold of $V$ containing all the integral points of $\Sigma$, then $\Sigma^{\prime}=\left\{\left.f\right|_{J^{1}\left(U, V_{1}\right)}: f\right.$ in $\left.\Sigma\right\}$ is an admissible restriction of $\Sigma$ to $V_{1}$. Then $\Sigma^{\prime}$ and $\Sigma$ have the same solutions.

Proposition 7. If $\Sigma^{\prime}$ on $V_{1}$ is an admissible restriction of $\Sigma$ on $V$, then at every integral point $p, \Sigma$ and $\Sigma^{\prime}$ have the same $(p-1)$-characteristics.

Proof. Let $\left(x^{i}, z^{\lambda}, u^{\pi}\right)$ be local coordinates on $V$ with $V_{1}$ defined by $u^{\pi}=0$, $\pi=1, \ldots, \pi_{t}$. Then the system $\Sigma$ " obtained by adding the $u^{\pi}$ to $\Sigma$ is equivalent to $\Sigma$ and so has the same characteristics. Hence $\partial_{i} u^{\pi}$ is in $\Sigma^{\prime \prime}$, and we may write the functions of $\Sigma^{\prime \prime}$ in the form $A_{\lambda}^{\alpha i} \partial_{i} z^{\lambda}+B^{\alpha}, \partial_{i} u^{\pi}, u^{\pi}$ so the functions of $\Sigma^{\prime}$ are $A_{\lambda}^{\alpha i} \sigma \partial_{i} z^{\lambda}+B^{\alpha} \sigma$, where $\sigma$ is the imbedding of $V_{1}$ into $V$. It follows that at any integral point of $V_{1}$ and for any $\alpha_{i}$ in $R$, rank $\Sigma_{f}^{\prime}=\operatorname{rank} \Sigma_{f}^{\prime \prime}-\pi_{t}$.

Proposition 8. If $\Sigma^{\prime}$ is a pp of $\Sigma$, then $P \Sigma$ is the pp of an admissible restriction of $\Sigma^{\prime}$.

Proof. Let $\sigma_{0}$ be defined by $K_{\lambda}^{\pi j} \partial_{j} z^{\lambda}+L^{\pi}$, where by condition (3) of Definition 3 the rank of $K_{\lambda}^{\pi j}$ is constant. It is thus possible locally, by a linear change of coordinates $u^{\pi}$, to write $\sigma_{0}$ in the form $u^{\pi}=K_{\lambda}^{\pi j} \partial_{j} z^{\lambda}, \pi=1, \ldots, \pi_{s} ; u^{\pi}=L^{\pi}$, $\pi=\pi_{s+1}, \ldots, \pi_{t}$, where $K_{\lambda}^{\pi j} \partial_{j} z^{\lambda}$ are linearly independent in $\partial_{j} z^{\lambda}$.

Let $\Sigma^{\prime \prime}$ be the admissible restriction to the submanifold defined by $u^{\pi}=L^{\pi}$. On $V_{0}=J^{1}(U, V)$ choose new coordinates $\left(x^{i}, z, u^{n}\right), 1 \leqq \pi \leqq p m$, where $u^{\pi}=K_{\lambda}^{\pi j} p_{j}^{\lambda}$, $1 \leqq \pi \leqq \pi_{s}, u^{\pi}=H_{\lambda}^{\pi j} p_{j}^{\lambda}, \pi>\pi_{s}$. Then $P \Sigma$ contains all the functions in $\Sigma^{\prime}$ by condition (2) of Definition 3 together with functions $u^{\pi}-H_{\lambda}^{\pi j} \partial_{j} z^{\lambda}$ and satisfies the conditions of Proposition 5.

2. Negative results. We next show by examples that the $(p-1)$-characteristics are not absolute invariants in general. (It is later proved that they are invariants in the special case of determined systems.) It is shown that even for determined systems the $(p-h)$-characteristics for $h \geqq 2$ need not be absolute invariants.

EXAMPLE 1. Here $U=R^{2}, V=U \times R^{2}$ and $\Sigma$ is generated by the single function $\partial_{2} z^{1}-\partial_{1} z^{1}-z^{2}$. Then $\Sigma_{f}$ contains only $\left(\alpha_{2}-\alpha_{1}\right) \xi^{1}$ and has rank 1 except when $\alpha_{2}-\alpha_{1}=0$. This defines the characteristic subspace of vectors $a\left(\partial / \partial x^{1}-\partial / \partial x^{2}\right)$. Let $V_{1}$ be $V \times R$ and $\Sigma^{\prime}$ be generated by $\partial_{2} z^{1}-\partial_{1} z^{1}-z^{2}, u^{1}-\partial_{1} z^{1}, \partial_{2} u^{1}-\partial_{1} u^{1}-\partial_{1} z^{2}$. This system is involutive and satisfies the hypotheses of Proposition 5 , but $\Sigma_{f}^{\prime}$ is generated by $\left(\alpha_{2}-\alpha_{1}\right) \xi^{1}, \alpha_{1} \xi^{1},\left(\alpha_{2}-\alpha_{1}\right) \eta-\alpha_{1} \xi^{2}$ which has rank 2 for every $\left(\alpha_{1}, \alpha_{2}\right)$ $\neq(0,0)$. Thus $\Sigma^{\prime}$ is a pp of $\Sigma$ having no characteristics.

EXAMPLE 2. Consider the over-determined system $\Sigma$ on $R^{3} \times R^{2}$ generated by $\partial_{3} z^{1}, \partial_{3} z^{2}, \partial_{2} z^{1}-\partial_{1} z^{1}-z^{2}$. The 1-dimensional characteristics are determined by the forms $\alpha_{3} \xi^{1}+\beta_{3} \eta^{1}, \alpha_{3} \xi^{2}+\beta_{3} \eta^{2}, \alpha_{2} \xi^{1}+\beta_{2} \eta^{1}-\alpha_{1} \xi^{1}-\beta_{1} \eta^{1}$. These forms have rank less than 3 only when $\alpha_{3}\left(\beta_{2}-\beta_{1}\right)=\beta_{3}\left(\alpha_{2}-\alpha_{1}\right)$. 
Let $\Sigma^{\prime}$ be obtained on $R^{3} \times R^{2} \times R$ by adding to $\Sigma$ the functions $u-\partial_{1} z^{1}, \partial_{3} u$, $\partial_{2} u-\partial_{1} u-\partial_{1} z^{2}$ which is involutive and a pp of $\Sigma$ by Proposition 5 . The additional forms for $\Sigma^{\prime}$ are $\alpha_{1} \xi^{1}+\beta_{1} \eta^{1}, \alpha_{3} \psi+\beta_{3} \phi, \alpha_{2} \psi+\beta_{2} \phi-\alpha_{1} \psi-\beta_{1} \phi-\alpha_{1} \xi^{2}-\beta_{1} \eta^{2}$ which altogether have rank 5. When $\alpha_{1}=\beta_{3}=0, \beta_{1}=\alpha_{3}=\beta_{2}=1$, and $\alpha_{2}$ is arbitrary, this determines a 1-characteristic of $\Sigma$ which is not a 1-characteristic of $\Sigma^{\prime}$.

3. Determined systems. Here $\Sigma$ is an involutive system on $V$ which is nonsingular, and $V$ is connected. Let $m=\operatorname{dim} V-\operatorname{dim} U$.

Definition 4. The system $\Sigma$ is $k$-determined if $k \geqq 1$ and $r_{p}=r_{p-1}=\cdots=r_{p-k+1}$ $=m$, but $r_{p-k}<m$.

REMARK. Systems are usually called "determined" when $r_{p}=m$ and $r_{h}=0$ for $h<p$. Such a property is not preserved under even total prolongations, while the above definition will be shown to be an absolute invariant. E. Cartan called $k$-determined systems "determined".

THEOREM 1. Let $\Sigma$ be a $k$-determined system generated near $\left(x_{0}, z_{0}\right)$ by

$$
\begin{aligned}
\theta_{r}^{\lambda} & =\partial_{r} z^{\lambda}-\sum_{a=1}^{p-k} A_{r \mu}^{\lambda a} \partial_{a} z^{\lambda}-B_{r}^{\lambda}, & & r=p-k+1, \ldots, p, \\
\phi^{\alpha} & =\sum_{a=1}^{p-k} C_{\lambda}^{\alpha a} \partial_{a} z^{\lambda}+D^{\alpha}, & \alpha & =1, \ldots, \alpha_{t} .
\end{aligned}
$$

Let $\alpha_{1}, \ldots, \alpha_{p-k}$ be in $R$. If we regard $A_{r}=\left(\sum_{a=1}^{p-k} A_{r \mu}^{\lambda a}\left(x_{0}, z_{0}\right) \alpha_{a}\right)$ as a matrix of linear transformations on the vector space $Z$ of m-rowed column vectors over $R$, and if $C$ is the subspace of $X$ spanned by $\left(\sum_{a=1}^{p-k} C_{\lambda}^{\alpha a}\left(x_{0}, z_{0}\right) \alpha_{a}\right), \alpha=1, \ldots, \alpha_{t}$, then

(a) $C$ is invariant under each $A_{r}$, and

(b) $A_{r} A_{s}=A_{s} A_{r}$ on $Z / C, p-k+1 \leqq r, s \leqq p$.

Proof. By involutiveness, agreeing that $a, b$ run from 1 to $p-k$,

$$
\partial_{p-k+h}^{*} \Sigma_{p-k} \subset \sum_{a=1}^{p-k} \partial_{a}^{*} \Sigma_{p-k+h}+\Sigma_{p-k+h}
$$

so

$$
\partial_{r}^{*} \phi^{\alpha}=R_{\lambda}^{\alpha s a} \partial_{a}^{*} \theta_{s}^{\lambda}+H_{\beta}^{\alpha a} \partial_{a}^{*} \phi^{\beta}+S_{\lambda}^{\alpha s} \theta_{s}^{\lambda}+T_{\beta}^{\alpha} \phi^{\beta} .
$$

Comparing coefficients of $\partial_{s a} z^{\lambda}, \delta_{r}^{s} C_{\lambda}^{\alpha a}=R_{\lambda}^{\alpha s a}$, and comparing coefficients of $\partial_{a b} z^{\lambda}$,

$$
0=-C_{\mu}^{\alpha a} A_{r \lambda}^{\mu b}-C_{\mu}^{\alpha b} A_{r \lambda}^{\mu a}+H_{\beta}^{\alpha a} C_{\lambda}^{\beta b}+H_{\beta}^{\alpha b} C_{\lambda}^{\beta a},
$$

which proves (a).

For $r<s, \partial_{s}^{*} \theta_{r}^{\lambda}$ is in $\partial_{1}^{*} \Sigma_{s}+\cdots+\partial_{r}^{*} \Sigma_{s}+\Sigma_{s}$, and so is

$$
\begin{aligned}
\partial_{s}^{*} \theta_{r}^{\lambda}-\partial_{r}^{*} \theta_{s}^{\lambda}+A_{r \mu}^{\lambda a} \partial_{a}^{*} \theta_{s}^{\mu}-A_{s \mu}^{\lambda a} \partial_{a}^{*} \theta_{r}^{\mu} \\
=-A_{r \mu}^{\lambda a} A_{s \nu}^{\mu b} \partial_{a b} z^{\nu}+A_{s u}^{\lambda a} A_{r v}^{\mu b} \partial_{a b} z^{\nu}+\text { lower order terms } \\
=\sum_{\sigma=p-k+1}^{s} \sum_{i=1}^{r} R_{\mu}^{\lambda \sigma i}\left(\partial_{i \sigma} z^{\mu}-A_{\sigma v}^{\mu a} \partial_{a i} z^{\nu}\right)+\sum_{i=1}^{r} H_{\alpha}^{\lambda a} C_{\mu}^{\alpha a} \partial_{i a} z^{\mu} .
\end{aligned}
$$


Hence $R_{\mu}^{\lambda \sigma i}=0$ if $\sigma>r$,

$$
\begin{gathered}
R_{\mu}^{\lambda \sigma \tau}+R_{\mu}^{\lambda \tau \sigma}=0 \text { for } p-k<\sigma, \tau \leqq r, \\
R_{\mu}^{\lambda \sigma a}-\sum_{\tau=p-k+1}^{r} R_{v}^{\lambda \tau \sigma} A_{\tau \mu}^{v a}+H_{\alpha}^{\lambda \sigma} C_{\mu}^{\alpha a}=0, \text { for } p-k<\sigma \leqq r,
\end{gathered}
$$

and

$$
-A_{r v}^{\lambda a} A_{s \mu}^{v b}+A_{s v}^{\lambda a} A_{r \mu}^{\nu b}-A_{r v}^{\lambda b} A_{s \mu}^{v a}+A_{s v}^{\lambda b} A_{r \mu}^{v a}=-R_{v}^{\lambda \sigma a} A_{\sigma \mu}^{v b}-R_{v}^{\lambda \sigma b} A_{\sigma \mu}^{v a}+H_{\alpha}^{\lambda a} C_{\mu}^{\alpha b}+H_{\alpha}^{\lambda b} C_{\mu}^{\alpha a} .
$$

The result follows using (1), (2) and (a).

Proposition 9. The $k$-determined system $\Sigma$ has at $\left(x_{0}, z_{0}\right) a(p-1)$-characteristic defined by $\alpha_{j}$ if and only if the following equivalent conditions are satisfied:

(a) There exist $\xi^{1}, \ldots, \xi^{m}$ in $R$, not all 0 , such that $\alpha_{r} \xi^{\lambda}=A_{r \mu}^{\lambda a}\left(x_{0}, z_{0}\right) \alpha_{a} \xi^{\mu}$, $r=p-k+1, \ldots, p ; \lambda=1, \ldots, m$, and $C_{\lambda}^{\alpha a}\left(x_{0}, z_{0}\right) \alpha_{a} \xi^{\lambda}=0, \alpha=1, \ldots, \alpha_{t}$;

(b) $\alpha_{r}$ is an eigenvalue of $A_{r}=\left(A_{r \mu}^{\lambda a}\left(x_{0}, z_{0}\right) \alpha_{a}\right)$ on $Z / C \neq 0$, and there is a common eigenvector $\xi^{*}$ of $A_{r}^{*}$, the adjoint linear transformation on $Z^{*}$, which vanishes on $C$.

Proof. $\Sigma_{f}$ has maximal rank $m$ when $\alpha_{1}=\cdots=\alpha_{p-1}=0$. The $\alpha_{j}$ are characteristic when the rank is less than $m$, which is the condition that the equations in (a) have a nontrivial solution. The exact sequence $0 \rightarrow C \rightarrow Z \rightarrow Z / C \rightarrow 0$ has dual $0 \leftarrow C^{*} \stackrel{\lambda}{\leftarrow} Z^{*} \leftarrow(Z / C)^{*} \leftarrow 0$, where $\lambda$ restricts to $C$. The $\xi^{\lambda}$ found in (a) define an element of $Z^{*}$ which belongs to $(Z / C)^{*}$ and is an eigenvector of all $A_{r}^{*}$ on $(Z / C)^{*}$ belonging to $\alpha_{r}$. Hence $\alpha_{r}$ is an eigenvalue of $A_{r}$ on $Z / C \neq 0$.

THEOREM 2. Let $\Sigma$ be the $k$-determined system generated by $\theta_{r}^{\lambda}, \phi^{\alpha}$ of Theorem 1 . Let $\Sigma^{\prime}$ be a pp of $\Sigma$ for which $x^{1}, \ldots, x^{p}$ are nonsingular near $\left(x_{0}, z_{0}, u_{0}\right)$. Let $x^{i}, z^{\lambda}, u^{\pi}$ be fibred coordinates for $\Sigma^{\prime}$ and suppose $\Sigma^{\prime}$ contains $u^{\pi}-K_{\lambda}^{\pi a} \partial_{a} z^{\lambda}-L^{\pi}=\psi^{\pi}$. Then

(a) $\Sigma^{\prime}$ is $k$-determined and contains

$$
\partial_{r} u^{\pi}-E_{\rho}^{\pi a} \partial_{a} u^{\rho}-F_{\lambda}^{\pi a} \partial_{a} z^{\lambda}-G^{\pi}, \quad \pi=1, \ldots, \pi_{t} ; \quad r=p-k+1, \ldots, p ;
$$

(b) at each point $\left(x_{0}, z_{0}, u_{0}\right)$ and for each $\alpha_{a}$ in $R,\left(K_{\lambda}^{\pi a}\left(x_{0}, z_{0}\right) \alpha_{a}\right)$ together with $\left(C_{\lambda}^{\alpha a}\left(x_{0}, z_{0}\right) \alpha_{a}\right)$ span as m-rowed column vectors a subspace $K \supset C$ of $Z$ which is invariant under all $A_{r}$, and

(c) $\left.A_{r}\right|_{(K / C)}$ is given by the matrix $\left(E_{\rho}^{\pi a}\left(x_{0}, z_{0}, u_{0}\right) \alpha_{a}\right)$.

Proof. By involutiveness, $\partial_{r}^{*} \psi^{\pi}+K_{\lambda}^{\pi a} \partial_{a}^{*} \theta_{r}^{\lambda}=-K_{\lambda}^{\pi a} A_{r \mu}^{\lambda b} \partial_{a b} z^{\mu}+$ terms of lower order is in $\partial_{1}^{*} \Sigma_{r}^{\prime}+\cdots+\partial_{p-k}^{*} \Sigma_{r}^{\prime}+\Sigma_{r}^{\prime}$. Now by Proposition 3 the only functions in $\partial_{a}^{*} \Sigma_{r}^{\prime}$ which are independent of $\partial_{i j} u^{\pi}$ are $\psi^{\pi}, \partial_{a}^{*} \psi^{\pi}$ and the functions in $\partial_{a}^{*} \Sigma_{r}$. Of the functions $\partial_{a}^{*} \theta_{r}^{\lambda}, \partial_{a}^{*} \phi^{\alpha}$ of $\partial_{a}^{*} \Sigma_{r}$, only $\partial_{a}^{*} \phi^{\alpha}$ are independent of $\partial_{a r} z^{\lambda}$. Hence

$$
\partial_{r}^{*} \phi^{\pi}+K_{\lambda}^{\pi a} \partial_{a}^{*} \theta_{r}^{\lambda}-E_{\rho}^{\pi a} \partial_{a}^{*} \psi^{\rho}-H_{\alpha}^{\pi a} \partial_{a}^{*} \phi^{\alpha}=\partial_{r} u^{\pi}-E_{\rho}^{\pi a} \partial_{a} u^{\rho}-F_{\lambda}^{\pi a} \partial_{a} z^{\lambda}-G^{\pi}
$$

is in $\Sigma_{r}^{\prime}$, proving (a). If in the last equation we compare coefficients of $\partial_{a b} z^{\lambda}$, we find

$$
0=-K_{\lambda}^{\pi a} A_{r \mu}^{\lambda b} \partial_{a b} z^{\mu}-E_{\rho}^{\pi a} K_{\lambda}^{\rho b} \partial_{a b} z^{\lambda}-H_{\alpha}^{\pi a} C_{\lambda}^{\alpha b} \partial_{a b} z^{\lambda} .
$$

This proves (b) and (c). 
COROLlARY. The property of being $k$-determined is an absolute invariant.

Proposition 10. Let $B_{1}, \ldots, B_{h}$ be a set of commuting complex $m \times m$ matrices: $B_{i} B_{j}=B_{j} B_{i}$. Let $K$ be a subspace of the vector space $Z$ of m-rowed complex column vectors which is invariant under all $B_{j}$. Suppose that $\eta$ is in $K^{*}$ and satisfies $\eta\left(\left.B_{h}\right|_{K}\right)^{*}$ $=0$, all $h$. Then there exists $\xi \neq 0$ in $Z^{*}$ such that $\xi B_{h}^{*}=0$, all $h$. (Here $B_{h}^{*}$ denotes the adjoint.)

Proof. Assume $Z / K$ contains no subspace invariant under all $B_{h}$. If $v$ is an eigenvector of $B_{h}$ on $Z / K$ belonging to $\lambda_{h}$, then $B_{h}\left(B_{k} v\right)=B_{k}\left(B_{h} v\right)=\lambda_{h} B_{k} v$, so the space of eigenvectors of $B_{h}$ belonging to $\lambda_{h}$ is invariant, hence spans $Z / K$. Choosing a basis $v^{1}, \ldots, v^{s}$ of $K$ and $v^{s+1}, \ldots, v^{m}$ of $Z$, we have

$$
\begin{array}{ll}
B_{h l} v^{i}=\sum_{j=1}^{s} C_{h j}^{i} v^{j} & \text { for } 1 \leqq i \leqq s, \\
B_{h} v^{\alpha}=\lambda_{h} v^{\alpha}+D_{h i}^{\alpha} v^{i} & \text { for } s<\alpha \leqq m .
\end{array}
$$

Supposing that $1 \leqq i, j \leqq s, s<\alpha, \beta \leqq m$, commutativity implies

$$
\lambda_{h} D_{k i}^{\alpha}+D_{h j}^{\alpha} C_{k i}^{j}=\lambda_{k} D_{h i}^{\alpha}+D_{k j}^{\alpha} C_{h i}^{j} \text {. }
$$

Let $\xi_{1}, \ldots, \xi_{m}$ be a dual basis to $v^{1}, \ldots, v^{m}$, so $\eta=y^{i} \xi_{i}$ satisfies $C_{h j}^{i} y^{j}=0$, $1 \leqq i \leqq s$. If some $\lambda_{h}=0,\left(^{*}\right)$ implies $\lambda_{k} D_{h i} y^{i}=0$, hence for any $y^{\alpha}, \xi=y^{i} \xi_{i}+y^{\alpha} \xi_{\alpha}$ satisfies $\xi B_{h}^{*}=y^{i} C_{h i}^{j} \xi_{j}+y^{i} D_{h i}^{\alpha} \xi_{\alpha}+\lambda_{h} y^{\alpha} \xi_{\alpha}$ so the extension of $\eta$ to $Z^{*}$ defined by $y^{\alpha}=0$ satisfies $\xi B_{h}^{*}=0$. If all $\lambda_{h} \neq 0$, then $\xi B_{h}^{*}=0$ if and only if $\lambda_{h} y^{\alpha}+y^{i} D_{h i}^{\alpha}=0$, i.e., $y^{\alpha}=-\left(1 / \lambda_{h}\right) y^{i} D_{h i}^{\alpha}$. Equation $\left(^{*}\right)$ guarantees that this condition is independent of $h$.

In general we choose $Z^{\prime}$ a smallest invariant subspace of $Z$ containing $K$ and use the above argument to extend $\eta$ to $Z^{\prime *}$, then repeat inductively with $Z^{\prime}$ instead of $K$.

THEOREM 3. If $\Sigma^{\prime}$ on $V_{1}$ is a nonsingular partial prolongation of a nonsingular system $\Sigma$ on $V$ where $\Sigma$ is $k$-determined and $\pi_{1}: V_{1} \rightarrow V$ is the fibration, then any $(p-1)$-characteristic of $\Sigma^{\prime}$ at $v_{1}$ in $V_{1}$ is a $(p-1)$-characteristic of $\Sigma$ at $\pi_{1}\left(v_{1}\right)$.

Proof. Assume $\Sigma$ generated in local fibred coordinates $\left(x^{i}, z^{\lambda}\right)$ by

$$
\begin{array}{ll}
\theta_{r}^{\lambda}=\partial_{r} z^{\lambda}-A_{r \mu}^{\lambda a} \partial_{a} z^{\mu}-B_{r}^{\lambda}, & r=p-k+1, \ldots, p, \\
\phi^{\alpha}=C_{\lambda}^{\alpha a} \partial_{a} z^{\lambda}+D^{\alpha}, & \alpha=1, \ldots, m,
\end{array}
$$

where $1 \leqq a, b \leqq p-k$, and suppose $\Sigma^{\prime}$ is generated by these forms together with

$$
\begin{gathered}
\psi^{\pi}=u^{\pi}-K_{\lambda}^{\pi a} \partial_{a} z^{\lambda}-L^{\pi}, \\
\partial_{r} u^{\pi}-E_{r \rho}^{\pi a} \partial_{a} u^{\rho}-F_{r \lambda}^{\pi a} \partial_{a} z^{\lambda}-G_{r}^{\pi}, \\
R_{\pi}^{\gamma a} \partial_{a} u^{\pi}+S_{\lambda}^{\gamma a} \partial_{a} z^{\lambda}+T^{\gamma}, \\
\pi=1, \ldots, \pi_{t} ; r=p-k+1, \ldots, p ; \gamma=1, \ldots, \gamma_{t} .
\end{gathered}
$$


The forms of $\Sigma_{f}^{\prime}$ are generated at $\left(x_{0}, z_{0}, u_{0}\right)$ by

$$
\begin{aligned}
& \left(\Sigma_{f}\right), \quad\left(\alpha_{r} \delta_{\mu}^{\lambda}-A_{r \mu}^{\lambda a}\left(x_{0}, z_{0}\right) \alpha_{a}\right) \xi^{\mu}, \quad C_{\lambda}^{\alpha a}\left(x_{0}, z_{0}\right) \alpha_{a} \xi^{\lambda}, \\
& K_{\lambda}^{\alpha a}\left(x_{0}, z_{0}\right) \alpha_{a} \xi^{\lambda}, \\
& \left(\alpha_{r} \delta_{\mu}^{\lambda}-E_{r \rho}^{\pi a}\left(x_{0}, z_{0}, u_{0}\right) \alpha_{a}\right) \eta^{\rho}-F_{r \lambda}^{\pi a}\left(x_{0}, z_{0}, u_{0}\right) \alpha_{a} \xi^{\lambda}, \\
& R_{\pi}^{\gamma a}\left(x_{0}, z_{0}, u_{0}\right) \alpha_{a} \eta^{\pi}+S_{\lambda}^{\gamma a}\left(x_{0}, z_{0}, u_{0}\right) \alpha_{a} \xi^{\lambda} .
\end{aligned}
$$

Assume $\left(\alpha_{j}\right)$ define a $(p-1)$-characteristic of $\Sigma^{\prime}$ at $\left(x_{0}, z_{0}, u_{0}\right)$. Then there exist $\xi^{\lambda}, \eta^{\pi}$ in $R$, not all zero, which annihilate $\Sigma_{f}^{\prime}$, by Proposition 9 . By the same proposition, if some $\xi^{\lambda} \neq 0$, then the $\xi^{\lambda}$ annihilate the forms of $\Sigma_{f}$, hence $\left(\alpha_{j}\right)$ define a $(p-1)$-characteristic of $\Sigma$ at $\left(x_{0}, z_{0}\right)$. Assume, then, that all $\xi^{\lambda}=0$. In particular, $\left(\alpha_{r} \delta_{\rho}^{\pi}-E_{r \rho}^{\pi a}\left(x_{0}, z_{0}, u_{0}\right)\right) \eta^{\rho}=0$. If $B_{r}=\delta_{\mu}^{\lambda}-A_{r \mu}^{\lambda a}\left(x_{0}, z_{0}\right) \alpha_{a}$ on $Z / C$, then $\left.B_{r}\right|_{K / C}$ is given by the matrix $\delta_{\rho}^{\pi}-E_{r o}^{\pi a}\left(x_{0}, z_{0}, u_{0}\right) \alpha_{a}$ by Theorem 2 . By Theorem $1, B_{r} B_{s}=B_{s} B_{r}$. If we consider these $B_{r}$ on $C^{n}$ instead of $R^{n}, n=\pi_{t}$, Proposition 10 guarantees the existence of complex $\xi^{\lambda}$, not all zero, satisfying $\xi B_{r}=0$ for all $r$. Taking the real or imaginary parts, we obtain real $\xi_{1} \neq 0$ in $(Z / C)^{*}$. This $\xi_{1}$ corresponds to $\xi$ in $Z^{*}$ which is zero on $C$ by the exact sequence $0 \rightarrow(Z / C)^{*} \rightarrow Z^{*} \rightarrow C^{*} \rightarrow 0$.

THEOREM 4. If $\Sigma^{\prime}$ is a partial prolongation of $\Sigma$ satisfying the conditions of Theorem 3 , then the $(p-1)$-characteristics of $\Sigma^{\prime}$ at $v_{1}$ coincide with those of $\Sigma$ at $\pi_{1}\left(v_{1}\right)$.

Proof. By Proposition $8 P \Sigma$ is a pp of an admissible restriction of $\Sigma^{\prime}$, and we apply Theorem 3 to $P \Sigma$ and $\Sigma^{\prime}, \Sigma^{\prime}$ and $\Sigma$, and then use Proposition 6 .

\section{REFERENCES}

1. E. Cartan, "Sur l'équivalence absolue de certains systèmes d'équations différentielles et sur certaines familles de courbes" in Oeuvres complètes, Partie II, vol. 2, Gauthier-Villars, Paris, 1953, pp. 1133-1168.

2. R. Hermann, E. Cartan's geometric theory of partial differential equations, Advances in Math. 1 (1965), 265-317.

3. H. H. Johnson, Absolute equivalence of exterior differential systems, Illinois J. Math. 10 (1966), 407-411.

4. - The noninvariance of hyperbolicity in partial differential equations, Pacific J. Math. 22 (1967), 419-430.

5. M. Kuranishi, Lectures on involutive systems of partial differential equations, Publicaçōes da Sociedade de Matemática de São Paulo, São Paulo, 1967.

UNIVERSITY OF WASHINGTON, SEATtLE, Washington 